\title{
Contributions to High Resolution and In Situ Electron Microscopy
}

\author{
Robert Sinclair ${ }^{1}$, Yunzhi Liu ${ }^{1}$, Sangchul Lee ${ }^{1}$ and Ai Leen Koh ${ }^{1}$ \\ ${ }^{1 .}$ Department of Materials Science and Engineering, Stanford University, Stanford, CA, USA.
}

Among the many contributions by Professor H. Hashimoto to the advancement of transmission electron microscopy (TEM) were his works on high resolution imaging. He was one of the first researchers to show the atomic arrangements in a close-packed metal (gold), including those at crystal defects [1]. Indeed, using the imaging electron beam as a stimulus, he made continuous recordings of dislocation behavior at the atomic level which were quite remarkable at the time [2]. Notable also in those days were Iijima's and Smith's videos [3,4] of the rapid atomic rearrangements in small gold particles (which would be called "nanoparticles" today). Our own research then concentrated on semiconductor systems and we were able to record various defect reactions in cadmium telluride including dislocation glide and climb, and surface atomic rearrangements $[5,6]$.

While these papers demonstrated the capability of high resolution imaging to reveal phenomena at the atomic scale, the use of beam stimulation clearly did not have the control and reproducibility to study reactions as they would occur during materials processing, manufacturing or service. Accordingly, our own work followed a different path whereby exact control of the means of stimulation for in situ observations became central $[7,8]$, resulting in a series of studies documenting and interpreting material reactions at the atomic level under controlled conditions (e.g. [9-13]). For annealing experiments, we combined quantitative measurements of reaction kinetics, including Arrhenius-type analyses, with the direct observation of the atomic processes involved, in many cases checking the results against those of equivalent bulk material.

It is possibly less well-known that Professor Hashimoto also demonstrated that solid-gas reactions could be studied at the magnification and resolution afforded by TEM, albeit not at atomic resolution. Thus, his papers $[14,15]$ showing sub-10nm resolution up to pressures of 300 Torr have an important status in the field of environmental TEM (ETEM), as described in a recent historical review of the field [16]. Of course, ETEM has become much more sophisticated now, with the introduction of specially designed specimen holders $[17,18]$ or differentially pumped ETEM machines which can even achieve aberration-corrected resolution [19]. Our own recent work on the dissipation of field-emitting carbon nanotubes in an oxygen environment [20,21] is only one of many examples of solid-gas reactions which have been influenced by Hashimoto's early work.

Figure 1 shows a recent example of cross-section high resolution imaging to demonstrate the loss of strontium iridate, a potential catalyst material, following electrochemical testing in a sulfuric acid environment for six hours.

In summary, it can be said that Professor Hashimoto was a true pioneer of high resolution and in situ electron microscopy and that his tenacity, skill and foresight have inspired numerous researchers globally to attempt to follow in his footsteps [22]. 
References:

[1] H. Hashimoto et al, J. Phys. Soc. Japan, 42 (1977) p.1073.

[2] H. Hashimoto et al, Jpn J. Appl. Phys., 19 (1980) p. L1.

[3] S.Iijima, and T. Ichihashi, Phys. Rev. Lett., 56 (1986) p. 616.

[4] D.J. Smith et al, Science, 233 (1986) p. 872.

[5] R. Sinclair, T. Yamashita and F.A. Ponce, Nature, 290 (1981) p. 386.

[6] R. Sinclair et al, Nature, 298 (1982) p. 127.

[7] R. Sinclair and M.A. Parker, Nature, 322 (1986) p. 531.

[8] R. Sinclair et al, Acta Crystallogr. Sec. A, 44 (1988) p. 965.

[9] T.J. Konno and R. Sinclair, Philos. Mag. B, 71 (1995) p. 179.

[10] D.H. Ko and R. Sinclair, Ultramicroscopy, 54 (1994) p. 166.

[11] T.J. Konno and R. Sinclair, Materials Science Forum, 204-206 (1996) p. 749.

[12] K.-H. Min et al, Philos. Mag., 85 (2005) p. 2049.

[13] R. Sinclair, Mat. Res. Soc. Bull. 38 (2013) p. 1065.

[14] H. Hashimoto, K. Tanaka and E.Yoda, J. Electron. Microsc.,6 (1958) p.8.

[15] H. Hashimoto et al, Jpn. J. Appl. Phys., 7 (1968) p.946.

[16] A. L. Koh, S. C. Lee and R. Sinclair, in "Controlled Atmosphere Transmission Electron Microscopy - Principles and Practice", ed. T. W. Hansen and J. B. Wagner (Springer International Publishing Switzerland, 2016), p. 3.

[17] J.F. Creemer et al, Ultramicroscopy, 108 (2008) p.993.

[18] N. de Jonge and F.M. Ross, Nature Nanotech., 6 (2011) p.695.

[19] E.D. Boyes and P.L. Gai, Ultramicroscopy, 67 (1997) p.219.

[20] A.L. Koh et al, Nanoscale, 8 (2016) p.16405.

[21] A.L. Koh and R. Sinclair, Ultramicroscopy, 176 (2017) p. 132.

[22] The present research of our group relevant to this paper has been funded by the National Cancer Institute under Grant U54 CA151459 to the Center for Cancer Nanotechnology Excellence and Translation Diagnostics (CCNE-TD) and by the Toyota Research Institute.
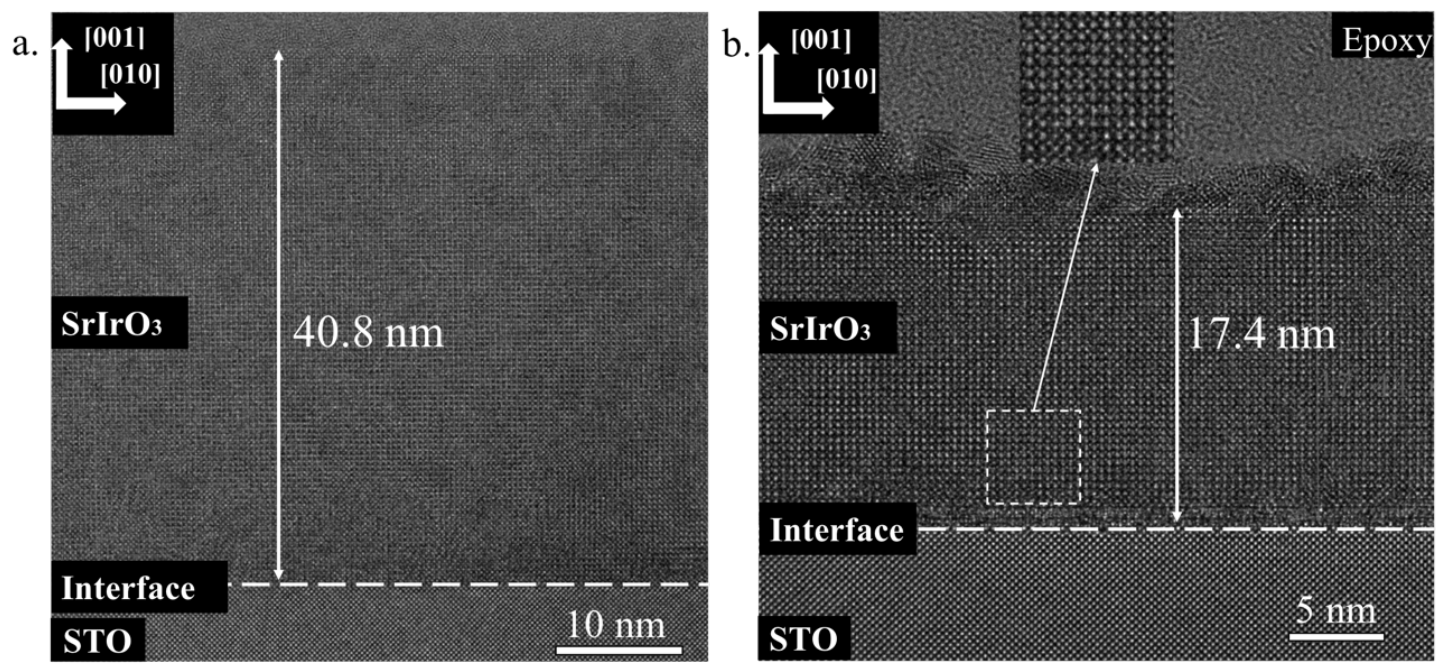

Figure 1. High resolution TEM images of (a) an as-deposited $\mathrm{SrIrO}_{3}$ thin film on a strontium titanate (STO) substrate and (b) the same thin film after an electrochemical test, showing the loss of strontium iridate in the electrochemical environment. 